\title{
Effect of camel milk on thymus and activation-regulated chemokine in autistic children: double-blind study
}

\author{
Shahid Bashir ${ }^{1,2}$ and Laila Y. Al-Ayadhi'
}

BACKGROUND: This study aimed to investigate the role of the effectiveness of camel milk (CM) (raw and boiled) on thymus and activation-regulated chemokine (TARC) serum levels and childhood autism rating scale (CARS) score in subjects with autism and compared to placebo group (cow milk).

METHODS: Forty-five subjects diagnosed with autism were randomly assigned to receive boiled CM for group I $(n=15)$, raw CM for group II $(n=15)$, and placebo for group III $(n=15)$ for 2 wk. Measures included changes in professionally completed CARS score and blood samples for TARC serum level were taken before and after milk consumption of $500 \mathrm{ml}$ per day in children's regular daily diet.

RESULTS: The serum levels of TARC decreased significantly $(P=0.004)$ in boiled $C M$ and in raw $C M$ group $(P=0.01)$ too, but no effect was observed $(P=0.68)$ in placebo group. Furthermore, significant improvements were observed in CARS score $(P=0.04)$ in raw CM group only. There were no significant relationships between the serum of TARC level and the CARS score, age, or gender for any group.

CONCLUSION: CM administered for $2 \mathrm{wk}$ significantly improved clinical measurements of autism severity and decreased serum level of TARC in autistic children, but subsequent studies are recommended.

A utism is a severe neurodevelopmental disorder that is characterized by impairment in verbal and nonverbal communication, imagination, reciprocal social interaction, and evidence of developmental delay within the first $3 \mathrm{y}$ of life (1-4). Immunological and environmental factors, such as diet, infection, and xenobiotics play critical roles in the development of autism (4-6). Over the years, research findings, especially from our lab, suggested possible involvement of altered immune system in the pathophysiology of autism spectrum disorder (ASD) $(7,8)$. Despite the clear unmet medical need, currently, there is no recognized effective comprehensive treatment (9).

Proinflammatory chemokines, such as monocyte chemotactic protein-1 and thymus and activation-regulated chemokine (TARC), along with cytokines, such as tumor necrosis factor $\alpha$, were consistently elevated in the brains of individuals with autism $(7,8)$. At critical times of infantile development, immune dysregulation may result in the release of immunemodulatory molecules, such as chemokines and cytokines, leading to altered neuronal development and neural function $(10,11)$. Furthermore, Ashwood and colleagues (2008) found that reduced levels of the modulatory cytokine, transforming growth factor- $\beta 1$ (TGF- $\beta 1$ ), in autistic children contributed to the dysregulation of adaptive behaviors and predisposal for autoimmune responses (12).

Milk is an important nutrient in human nourishment. In some communities, camels represent the most important source of this nutrient. Camel milk (CM) has emerged to have potential therapeutic effects in diseases such as diabetes $(13,14)$, hepatitis B (15), possibly certain symptoms accompanying autism enterocolitis, $H$. pylori infection and lactase deficiency might be cured with CM as well (16). Recently, some parents have been using camels' milk as a treatment in some children with ASD because camels' milk appears to help food allergies in some individuals $(17,18)$. CM's lactoferrin has very high levels of bactericidal and bacteriostatic properties against Gram-positive and Gram-negative bacteria (19), more than cow and human lactoferrin. CM contains various protective proteins, mainly enzymes which exert antibacterial and immunological properties (20). The fact that CM lacks $\beta$-lactoglobulin and a "new" $\beta$-casein (21), two powerful allergens in cow milk, makes the milk attractive for children suffering from milk allergies (22). Phylogenetic differences could be responsible for the failed recognition of camels' proteins by circulating IgEs and monoclonal antibodies (23). Children with severe food allergies improved rapidly with CM. CM with its unique properties, such as, high vitamin $\mathrm{C}$ levels, low fat content, and low molecular weight immunoglobulins, makes an ideal natural intervention method in ASD.

The hypothesis tested in the present study was that TARC act on their chemokine receptor type 4 (CCR4) receptors to enhance the recruitment and activation of T helper 2 cells with a subsequent production of type 2 cytokines that include interleukin (IL)-4, IL-5, IL-9, and IL-13 (24,25). CCR4 ligands have an important pathogenic role in inflammatory conditions such

\footnotetext{
'Department of Physiology, Autism Research and Treatment Center, Shaik AL-Amodi Autism Research Chair, Faculty of Medicine, King Saud University, Riyadh, Saudi Arabia; 2Division of Cognitive Neurology, Department of Neurology, Berenson-Allen Center for Noninvasive Brain Stimulation, Beth Israel Deaconess Medical Center, Harvard Medical School, Boston, Massachusetts. Correspondence: Laila Y. Al-Ayadhi (ayadh2@gmail.com)
} 
as allergy and some autoimmune diseases (26-28). TARC and their receptors have been implicated as functional mediators of immunopathology of autoimmune neuroinflammatory diseases $(29,30)$ and childhood autism rating scale (CARS) score which have a significant impact on behavior, cognition, socialization, and health/physical traits associated with an ASD diagnosis.

The present prospective, double-blind, placebo-controlled trial evaluated whether a standardized CM administered to patients diagnosed with an ASD on a daily basis for 2 wk would result in improved CARS score traits associated with an ASD diagnosis.

\section{RESULTS}

The general characteristics of the study participants and the results of the serum levels of TARC are depicted in Table 1 . No significant differences were observed between the CM (raw or boiled) and placebo groups with respect to age, gender, or ASD diagnostic status. There were no significant differences on serum levels of TARC at baseline between the randomization groups $(P=0.28)$ and on the CARS score between groups $(P=0.719)$.

Figure 1 summarizes the change in serum levels of TARC after the CM (raw or boiled) and placebo groups following $2 \mathrm{wk}$ of therapy. Changes in serum levels of TARC significantly decreased $(P=0.004, P=0.01$, for values see Table 2$)$ for $\mathrm{CM}$ (boiled and raw) group respectively. In contrast, no similar changes were observed ( $P=0.54$, for values see Table 2$)$ for

Table 1. Baseline characteristics of camel milk group (raw and boiled) and placebo (cow milk) cohorts at randomization

\begin{tabular}{lccc}
\hline & \multicolumn{2}{c}{ Camel milk group } & \\
\cline { 2 - 3 } & Raw & Boiled & Placebo group \\
\hline Age (year) & $7.1 \pm 3.8^{\mathrm{a}}$ & $6.8 \pm 4.1$ & $6.9 \pm 4.3$ \\
Gender $(n)$ & & & \\
Male & 13 & 14 & 13 \\
Female & 2 & 1 & 2 \\
\hline
\end{tabular}

aMean \pm SD.

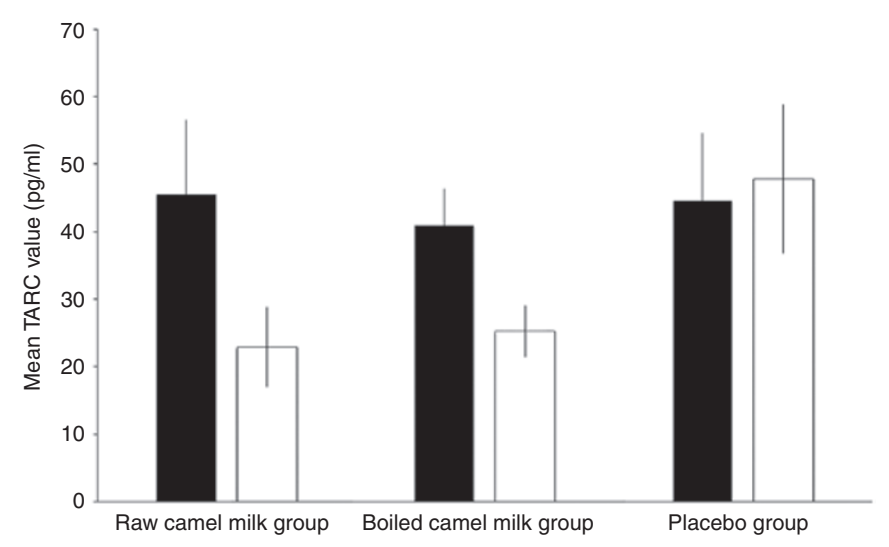

Figure 1. Serum levels of thymus and activation-regulated chemokine (TARC) before (black columns) and after (white columns) in camel milk group (raw and boiled) and placebo (cow milk) group of autistic children. placebo group. There was a significant difference $(P=0.04$, for values see Table 3) for CARS score from $37.13 \pm 5.3$ (mean \pm $\mathrm{SEM}$ ) to $33.8 \pm 2.7$ (mean \pm SEM) in raw CM group only. No significant correlation was found between serum TARC levels and CARS score for children with ASD for any group.

\section{DISCUSSION}

The present study is the first prospective, double-blind, placebo-controlled trial to evaluate the effects of $\mathrm{CM}$ therapy among subjects diagnosed with an ASD. In the present study, CM therapy (raw CM group) for $2 \mathrm{wk}$ among subjects diagnosed with an ASD significantly improved clinical measurements (CARS score) recorded by trained professional and parents of study subjects. Furthermore, CM therapy (raw and boiled) significantly decreased serum levels of TARC among patients diagnosed with an ASD. Finally, CM therapy was generally well tolerated with minimal adverse effects. The side effects in the children who did not tolerate the treatment well were irritability and/or stomach discomfort.

$\mathrm{CM}$, with its distinctive properties, could be a promising beneficial intervention approach in ASD. Mammalian antibodies composed of two identical $\mathrm{H}$-chains and two identical L-chains (31). Camel IgG antibodies are heavy-chain antibodies that lack the L-chain (31). The size of the antibodies is a major issue in the development of human immunotherapy. Camel antibodies are only one tenth of the size of human antibodies, which makes them natural nano-bodies $(31,32)$. Furthermore, the high content of vitamin $\mathrm{C}$ in $\mathrm{CM}$ gives it a string antioxidant property (33). Recent reports have demonstrated higher oxidative stress status in ASD subjects compared to normally developing controls (34-36), which makes CM an ideal antioxidant food for ASD subjects. In addition, those unique properties of CM most probably reduced TARC synthesis and secretion, and consequently, reducing the neuroimflammation and the autoimmune reaction, leading to improved behavior, reflected on improved CARS scoring results.

Table 2. Serum levels of TARC results for camel milk group (raw, boiled) and placebo (cow milk) groups

\begin{tabular}{lcc}
\hline & \multicolumn{2}{c}{ TARC $(\mathrm{pg} / \mathrm{ml})$} \\
\cline { 2 - 3 } & \multicolumn{1}{c}{ Before } & After \\
\hline Boiled camel milk group $(n=15)$ & $40.09 \pm 5.4^{\mathrm{a}}$ & $25.25 \pm 3.08^{*}$ \\
Raw camel milk group $(n=15)$ & $45.52 \pm 11$ & $22.86 \pm 5.9^{*}$ \\
Placebo group $(n=15)$ & $44.46 \pm 10.11$ & $47.8 \pm 11$ \\
\hline $\begin{array}{l}\text { TARC, thymus activation-regulated chemokine. } \\
\text { aMean } \pm \text { SD. * } \text { Significance level }(P=0.05) .\end{array}$
\end{tabular}

Table 3. Childhood autism rating scale scoring results for camel milk (raw, boiled) and placebo (cow milk) groups

\begin{tabular}{lll}
\hline & Before & After \\
\hline Boiled camel milk group $(n=15)$ & $38 \pm 5.4^{\text {a }}$ & $35 \pm 2.7$ \\
Raw camel milk group $(n=15)$ & $37 \pm 3.8$ & $32 \pm 2.6^{*}$ \\
Placebo group $(n=15)$ & $36 \pm 3$ & $33 \pm 3.4$ \\
\hline
\end{tabular}

aMean \pm SD. *Significance level $(P=0.05)$. 
However, a clear pattern has emerged over several studies that shows altered levels of immune mediators are associated with increased impairments in behaviors $(7,12,37,38)$ and suggests that a dysregulated immune response is related to behavioral and cognitive impairments in children with ASD. The elements such as zinc, copper, selenium, and iron are not likely to have influenced our results, since their amounts in CM and in cow milk are practically the same (39).

\section{Strengths/Limitations}

The main strength of the present study is the design as a prospective, double-blind, placebo-controlled trial. Every effort was made to ensure that the present study was truly doubleblind so that those evaluating study subjects, both trained professionals and parents, had no knowledge as to the treatment status of any particular study subject. Furthermore, the present study also attempted to minimize the effects of study drop-out for potential adverse reactions in the data, especially for CARS scores. These particular scoring measurements were conducted by the study investigators on each child regardless of whether or not they dropped-out from the study for potential adverse reactions. For each outcome measurement evaluated, the relative change for the parameter following $2 \mathrm{wk}$ of therapy in comparison to baseline was examined. As a result, potential variation between study subjects was minimized because each study subject served as his or her own control.

One of the potential limitations of the present study is the small sample size examined. The small sample size in the present study may have resulted in specific effects of CM therapy being missed because of lack of statistical power to detect significant changes between the CM (raw or boiled) and placebo groups. As a result, the observation of significant positive effects of CM therapy in the present study tends to argue that the observed effects represent genuine phenomena. The data from the present study provide the basis for a larger, more focused study on the promising elements.

A further potential limitation of the present study is the exact mechanism of action of CM was not elucidated from the present study. Finally, an additional potential limitation of the present study is the fact that the dose of CM used may not have been optimal. The dosing regimen of CM used in the present study was derived from pediatric nutrition, as the recommended starting dose for children.

\section{Conclusion}

Camels' immune systems are stronger than that of humans and the small immunoglobulins pass from the CM into the human blood. As immunoglobulins are found in CM throughout lactation, drinking milk will provide a "tool" for combating autoimmune diseases by rehabilitating the immune system rather than is depression.

In conclusion, the results of the present study suggest that CM therapy over the course of 2 wk of therapy significantly improved clinical measurements of ASD severity (CARS score). Furthermore, there were significant decrease levels of serum of TARC among the study subjects examined. Overall, the CM therapy was well tolerated. It is suggested that future studies further explore the biological basis for CM's mode of action at the cellular level in those patients diagnosed with an ASD who would most benefit from CM therapy.

\section{METHODS \\ Subjects}

A total of 45 subjects diagnosed with ASD, aged from 2 to $12 \mathrm{y}$ (40 males, 5 females), were recruited to the study. The study subjects have body weight between $12.8 \mathrm{~kg}$ and $42.6 \mathrm{~kg}$. None of the study subjects had previously received CM therapy. None of the study subjects had any change in therapy or treatment (including medications) within 1 mo prior to the study. Patients fulfilled the criteria for the diagnosis of autism according to the 4th edition of the Diagnostic and Statistical Manual of Mental Disorders (2). The study protocol received Institutional Review Board approval from King Khalid Hospital (King Saud University, Riyadh, Saudi Arabia). All parents signed a consent form and all received a copy.

\section{Clinical Assessment}

Autism Diagnostic Observation Schedule is a semistructured, standardized observational instrument to assess the social and communicative abilities of individuals with possible ASD. Items are scored from 0 (not abnormal) to 2 or 3 (most abnormal), and a diagnosis of autism or ASD is established if the individual assessed has scores higher than the established cut-off values in the communication domain, the social domain, and a sum of the two (40).

\section{Clinical Measure}

Childhood autism rating scale. Study participants were evaluated using CARS test conducted only by a single child psychiatrist who observed the subjects and interviewed the parent(s) and was unaware as to the treatment status of the subject. The CARS test is a 15-item behavioral rating scale (relating to people, emotional response, imitation, body use, object use, listening response, fear or nervousness, verbal communication, nonverbal communication, activity level, consistency of intellectual response, adaptation to change, taste, touch and smell response, and general impressions) developed to identify autism as well as to quantitatively describe the severity of the disorder. The CARS test is a well-established measure of autism severity (41). The internal consistency reliability alpha coefficient is 0.94 ; the interrater reliability correlation coefficient is 0.71 ; and the test-retest correlation coefficient is 0.88 (42). CARS scores have high criterion-related validity when compared to clinical ratings during the same diagnostic sessions, with a significant correlation of $0.84(42)$.

\section{Lab Testing}

Blood samples. After an overnight fasting, blood samples $(3 \mathrm{ml})$ were collected from subjects in both groups in plain test tubes. Blood samples were allowed to clot and then centrifuged at $3,000 \mathrm{rpm}$ to collect serum samples, which were stored frozen in a freezer at $-80^{\circ} \mathrm{C}$ until the time of analytical assays. The detail procedure has been described in our previous work $(7,8,43)$

Chemokine assay. Serum level of TARC was measured using a commercially available sandwich enzyme immunoassay (ELISA) kit from CUSABIO BIOTECH (Wuhan, China).

\section{Study Milk}

CM (boiled or raw) was supplied in a liquid preparation. The placebo (cow milk) group was identical in appearance. The recommended childhood starting dose of $500 \mathrm{ml}$ milk per day (half the total dose administered in the morning and half the total dose administered in the evening). Study subject-specific dosing instructions were placed on each liquid preparation provided to study subjects. Parents were asked to continue with the children's daily routines. They were not allowed to add or remove any interventions such as diet plans, supplements, or pharmacotherapies throughout the study period. 
Milk handling. Fresh CM was obtained from a trusted camel farm that ran regular routine veterinary checkups on the camels. After receiving the milk, microbiological screening tests were conducted on all milk batches to ensure that it was free of pathogens commonly found in raw $\mathrm{CM}$ (44). The pathological screenings were conducted to detect Campylobacter (KGaA, Darmstadt, Germany) Bacillus cereus enterotoxin, E. coli O157:H7, Listeria, Salmonella by GLISA rapid testing using the kits Singlepath Campylobacter, Duopath Cereus Enterotoxin (EMD chemicals), Reveal E. coli O157:H7, Salmonella, Listeria (Neogen), and B. Brucella (Anigen). Any batch-tested positive for the mentioned pathogens was immediately excluded from the study. CM supplied to group I was pasteurized by heating to $65^{\circ} \mathrm{C}$ for $15 \mathrm{~s}$, then removed, cooled in a ice pot initially and then stored in the freezer at $-80^{\circ} \mathrm{C}$. Milk supplied to group II was not heated to avoid losing beneficial nutrients and proteins (45). Frozen milk was supplied to patients using bisphenol-A-free freezer bottles and thawed on countertops as needed.

\section{Study Design}

This was a randomized, double-blind, placebo-controlled study. The study was conducted between 2011 and 2012. The study subjects were recruited through community contacts. The study protocol called for 36 subjects to receive CM (raw or boiled) and 18 study subjects to receive placebo (cow milk). A total of 54 subjects were recruited for the present study. Four subjects withdrew prior to randomization into $\mathrm{CM}$ or placebo groups. A total of 54 subjects were randomly assigned to receive CM (boiled or raw) or placebo, and of these, a total of 9 subjects ( 4 in boiled, 2 in raw in CM group and 3 in the placebo group) withdrew prior to successful completion of $2 \mathrm{wk}$ of therapy. Among the nine subjects withdrawing from the study prior to successful completion of $2 \mathrm{wk}$ of therapy, four subjects withdrew because of adverse reactions (two in raw CM group, one in boiled CM group and 1 in the placebo group), three subjects did not comply with the study protocol, and two was lost to follow-up with no known adverse reaction. In addition, study investigators monitored study subjects to ensure compliance and to monitor for potential adverse reactions.

\section{Prerandomization Phase}

Study subjects were seen for an initial screening where study investigators obtained information regarding demographics, formal diagnosis, age at diagnosis, age of apparent onset, information regarding delay or regression, any current medical issues, medications, bodyweight, and allergies on each study subject. A baseline CARS evaluation was performed by a child psychiatrist. In addition, blood samples were collected on each study subject at an autism research and treatment center draw station.

\section{Randomization Phase}

Following the initial screening and collection of labs, all study subjects started therapy within $30 \mathrm{~d}$ of baseline measurements. A study investigator, who did not perform any clinical measurements on study subjects, used a coin-flip to randomly assign study subjects to either the CM (raw or boiled) or placebo groups. Since there was a difference in sample size between the CM and placebo groups, the placebo group was filled with study subjects before the treatment group, so that the latter study subjects were all assigned to the CM group (raw or boiled). Study investigators in contact with the study subjects and the parents of study subjects were not informed of the treatment status (CM/placebo) of each study participant until all study subjects had completed the trial, and hence the assignment (CM/placebo) strategy used should not have revealed any information regarding the treatment status of any study participant to study investigators in contact with study subjects and the parents of study subjects. For the duration of the trial, any concomitant use of drugs/supplements were not changed as far as possible.

\section{Statistical Analysis}

The results were analyzed using the commercially available software package Statview (Abacus concepts, Berkley, CA). The data are presented as the means \pm SEM. The Mann-Whitney test was used for comparisons between data. The null hypothesis was that there would be no difference in the data distributions of the relative change in test results following $2 \mathrm{wk}$ of treatment in comparison to baseline measurements between study subjects receiving CM (raw or boiled) in comparison to placebo (cow milk). In addition, the relationship between the change in serum levels of TARC following $2 \mathrm{wk}$ of treatment in comparison to his or her baseline measurements, and the changes in specific outcome measurements (CARS scores) $2 \mathrm{wk}$ of treatment in comparison to his or her baseline measurements. Spearman's rank correlation coefficient " $r$ " was used to determine the relationship between variables. For all statistical tests performed in the present study, a two-tailed $P$ value $\leq 0.05$ was considered to be statistically significant.

\section{ACKNOWLEDGMENTS}

The authors thank Rana Zeina and Zakia Mohammed for their administrative help.

\section{STATEMENT OF FINANCIAL SUPPORT}

Work on this study was supported by grants from the King Abdulaziz City for Science and Technology (A-L-11-0808), and National Plane of Science and Technology Health Research program and Deanship of Scientific Research grant (RGP-VPP-216) from King Saud University, Saudi Arabia.

\section{REFERENCES}

1. Baird G, Simonoff E, Pickles A, et al. Prevalence of disorders of the autism spectrum in a population cohort of children in South Thames: the Special Needs and Autism Project (SNAP). Lancet 2006;368:210-5.

2. American Psychiatric Association. Diagnostic and Statistical Manual of Mental Disorders, 4th edn, Text Revision. Washington, DC, American Psychiatric Association, 2000.

3. Hollander E, Phillips A, King BH, et al. Impact of recent findings on study design of future autism clinical trials. CNS Spectr 2004;9:49-56.

4. Ivarsson SA, Bjerre I, Vegfors P, Ahlfors K. Autism as one of several disabilities in two children with congenital cytomegalovirus infection. Neuropediatrics 1990;21:102-3.

5. Edelson SB, Cantor DS. The neurotoxic etiology of the autistic spectrum disorder: A replicative study. Toxicol Ind Health 2000;16:239-47.

6. Fatemi SH, Earle J, Kanodia R, et al. Prenatal viral infection leads to pyramidal cell atrophy and macrocephaly in adulthood: implications for genesis of autism and schizophrenia. Cell Mol Neurobiol 2002;22:25-33.

7. Al-Ayadhi LY, Mostafa GA. Low plasma progranulin levels in children with autism. J Neuroinflammation 2011;8:111.

8. Al-Ayadhi LY, Mostafa GA. A lack of association between elevated serum levels of S100B protein and autoimmunity in autistic children. J Neuroinflammation 2012;9:54.

9. Gerlai J, Gerlai R. Autism: a large unmet medical need and a complex research problem. Physiol Behav 2003;79:461-70.

10. Plioplys AV. Intravenous immunoglobulin treatment of children with autism. J Child Neurol 1998;13:79-82.

11. Ashwood P, Wills S, Van de Water J. The immune response in autism: a new frontier for autism research. J Leukoc Biol 2006;80:1-15.

12. Cohly HH, Panja A. Immunological findings in autism. Int Rev Neurobiol 2005;71:317-41.

13. Agrawal RP, Jain S, Shah S, Chopra A, Agarwal V. Effect of camel milk on glycemic control and insulin requirement in patients with type 1 diabetes: 2-years randomized controlled trial. Eur J Clin Nutr 2011;65:1048-52.

14. Mohamad RH, Zekry ZK, Al-Mehdar HA, et al. Camel milk as an adjuvant therapy for the treatment of type 1 diabetes: verification of a traditional ethnomedical practice. J Med Food 2009;12:461-5.

15. Saltanat H, Li H, Xu Y, Wang J, Liu F, Geng XH. [The influences of camel milk on the immune response of chronic hepatitis B patients]. Xi Bao Yu Fen Zi Mian Yi Xue Za Zhi 2009;25:431-3.

16. Kushak RI, Lauwers GY, Winter HS, Buie TM. Intestinal disaccharidase activity in patients with autism: effect of age, gender, and intestinal inflammation. Autism 2011;15:285-94.

17. Shabo Y, Barzel R, Margoulis M, Yagil R. Camel milk for food allergies in children. Isr Med Assoc J 2005;7:796-8.

18. Shabo Y, Yagil R. Etiology of autism and camel milk as therapy. Int J Dis Human Dev 2005;4:67-70.

19. Borish LC, Steinke JW. 2. Cytokines and chemokines. J Allergy Clin Immunol 2003;111:Suppl 2:S460-75. 
20. Kappeler S, Farah Z, Puhan Z. Sequence analysis of Camelus dromedarius milk caseins. J Dairy Res 1998;65:209-22.

21. Beg OU, von Bahr-Lindström H, Zaidi ZH, Jörnvall H. Characterization of a camel milk protein rich in proline identifies a new beta-casein fragment. Regul Pept 1986;15:55-61.

22. Makinen-Kijunen S, Palosne T. A sensitive enzyme-linked immunosorbent assay for determination of bovine beta-lactoglobulin in infant feeding formulas and human milk. Allergy 1992;47:347-52.

23. Restani P, Gaiaschi A, Plebani A, et al. Cross-reactivity between milk proteins from different animal species. Clin Exp Allergy 1999;29:997-1004.

24. Kunkel EJ, Boisvert J, Murphy K, et al. Expression of the chemokine receptors CCR4, CCR5, and CXCR3 by human tissue-infiltrating lymphocytes. Am J Pathol 2002;160:347-55.

25. Bhowmick S, Chatterjee D, Chaudhuri K. Human epithelial cells stimulated with Vibrio cholerae produce thymic stromal lymphopoietin and promote dendritic cell-mediated inflammatory Th2 response. Int J Biochem Cell Biol 2012;44:1779-90.

26. Oliphant CJ, Barlow JL, McKenzie AN. Insights into the initiation of type 2 immune responses. Immunology 2011;134:378-85.

27. Machura E, Rusek-Zychma M, Jachimowicz M, Wrzask M, Mazur B, Kasperska-Zajac A. Serum TARC and CTACK concentrations in children with atopic dermatitis, allergic asthma, and urticaria. Pediatr Allergy Immunol 2012;23:278-84.

28. Abelius MS, Ernerudh J, Berg G, Matthiesen L, Nilsson LJ, Jenmalm MC. High cord blood levels of the T-helper 2-associated chemokines CCL17 and CCL22 precede allergy development during the first 6 years of life. Pediatr Res 2011;70:495-500.

29. Karpus WJ, Fife BT, Kennedy KJ. Immunoneutralization of chemokines for the prevention and treatment of central nervous system autoimmune disease. Methods 2003;29:362-8.

30. Karpus WJ, Ransohoff RM. Chemokine regulation of experimental autoimmune encephalomyelitis: temporal and spatial expression patterns govern disease pathogenesis. J Immunol 1998;161:2667-71.

31. Ashwood P, Enstrom A, Krakowiak P, et al. Decreased transforming growth factor betal in autism: a potential link between immune dysregulation and impairment in clinical behavioral outcomes. J Neuroimmunol 2008;204:149-53.

32. Rellini E, Tortolani D, Trillo S, Carbone S, Montecchi F. Childhood Autism Rating Scale (CARS) and Autism Behavior Checklist (ABC) correspondence and conflicts with DSM-IV criteria in diagnosis of autism. J Autism Dev Disord 2004;34:703-8.

33. Hamers-Casterman C, Atarhouch T, Muyldermans S, et al. Naturally occurring antibodies devoid of light chains. Nature 1993 ;363:446-8.

34. Al-Gadani Y, El-Ansary A, Attas O, Al-Ayadhi L. Metabolic biomarkers related to oxidative stress and antioxidant status in Saudi autistic children. Clin Biochem 2009;42:1032-40.

35. Frustaci A, Neri M, Cesario A, et al. Oxidative stress-related biomarkers in autism: systematic review and meta-analyses. Free Radic Biol Med 2012;52:2128-41.

36. Rose S, Melnyk S, Pavliv O, et al. Evidence of oxidative damage and inflammation associated with low glutathione redox status in the autism brain. Transl Psychiatry 2012;2:e134.

37. Grigorenko EL, Han SS, Yrigollen CM, et al. Macrophage migration inhibitory factor and autism spectrum disorders. Pediatrics 2008;122:e438-45.

38. Biber K, Vinet J, Boddeke HW. Neuron-microglia signaling: chemokines as versatile messengers. J Neuroimmunol 2008;198:69-74.

39. Patterson PH. Immune involvement in schizophrenia and autism: etiology, pathology and animal models. Behav Brain Res 2009;204:313-21.

40. Oosterling I, Roos S, de Bildt A, et al. Improved diagnostic validity of the ADOS revised algorithms: a replication study in an independent sample. J Autism Dev Disord 2010;40:689-703.

41. Chlebowski C, Green JA, Barton ML, Fein D. Using the childhood autism rating scale to diagnose autism spectrum disorders. J Autism Dev Disord 2010;40:787-99.

42. Schopler E, Reichler RJ, Renner BR. The Childhood Autism Rating Scale. Los Angeles, CA, Western Psychological Services, 1994:90025-1251.

43. Mostafa GA, Al-Ayadhi LY. The relationship between the increased frequency of serum antineuronal antibodies and the severity of autism in children. Eur J Paediatr Neurol 2012;16:464-8.

44. Eberlein V. Hygienic Status of Camel Milk in Dubai (United Arab Emirates) Under Two Different Milking Management Systems. München, Germany, Maximilians-Universität München, 2007. (http:// urn:nbn:de:bvb.)

45. Elagamy I. Effect of heat treatment on camel milk proteins with respect to antimicrobial factors: a comparison with cows' and buffalo milk proteins. Food Chemistry 2000;68:227-32. 